\title{
Approach towards optimal physiological T-cell-mediated immune response
}

Evaluation of: Kirak O, Frickel EV, Grotenbreg GM et al.: Transnuclear mice with predefined T cell receptor specificities against Toxoplasma gondii obtained via SCNT. Science 238, 243-248 (2010). Transgenic mice with defined T-cell receptor (TCR) specificity are useful for studying the involvement of T-cell-mediated immune responses to antigenic epitopes. However, these transgenic mice were not easily established. Their creation is time consuming due to several hindrances such as random integration of the $\alpha$ - and $\beta$-chains of the transgenic TCR and the presence of exogenous promoter, among others. These drawbacks explain why only a few TCR transgenic mice are available so far. Somatic cell nuclear transfer represents another means to generate mice with defined TCR specificity. Kirak et al. described an experimental model based on somatic cell nuclear transfer that allowed them to obtain mice harboring TCR specific to Toxoplasma gondii. The apparent ease in establishing this experimental model could lead to the generation of mice with defined T-cell immune responses against other antigens in the near future.

KEYWORDS: somatic cell nuclear transfer receptor transgenic mice

Stimulating a homogenous and efficient immune response against pathogens is not an easy task. This is due to unpredictable parameters such as the interplay between lymphoid subpopulations, mainly $\mathrm{T}$ helper and regulatory $\mathrm{T}$ cells and $\mathrm{T}$ and $\mathrm{B}$ lymphocytes, and the nature and the availability of the antigen $[1,2]$. Moreover, it is well known that an immune response against pathogens usually generates numerous CD4 and CD8 T-cell clones recognizing several naturally processed antigenic epitopes from the pathogen [3-5]. Thus, for enforcing and optimizing the immune response, transgenic mice with specifically rearranged T-cell receptors (TCRs) had been created $[6,7]$. These models allowed exploration of functional aspects relevant to single clones that are rare and difficult to study in wild-type organisms. It is worth noting that the small number of TCR transgenic mice so far obtained were essentially derived from T-cell clones/T hybridomas after in vitro culture. Nevertheless, these TCR transgenic mice have contributed to T-cell epitope characterization in the context of defined MHC-I/MHC-II that allows experiments to be carried out concerning amino acid substitutions and modifications of antigenic peptides into agonist and/or antagonist for reactive $T$ cells [8-10]. These studies were essential in giving insights into the regulation of the immune response in autoimmunity, graft rejection and tumor immunity.
T-cell-mediated immune response T-cell

\section{Summary of methods \& results}

In their work, Kirak et al. used somatic cell nuclear transfer (SCNT) from somatic immune cells to establish transgenic mice without introducing genetic modifications [11]. The method was applied to $\mathrm{CD} 8^{+}$effector $\mathrm{T}$ lymphocytes specific for Toxoplasma gondii, isolated from mice that had resolved an acute infection. These CD $8^{+} \mathrm{T}$ cells, specific for naturally processed T. gondii epitopes, in particular R7 (Ld-Tg-Rop7 161-169), G4 (Ld-Tg-Grad 107-115) and T57 (Kb-Tg-tgdO57 59-66), were thus used as donor cells for SCNT. For improving nuclear reprogramming, the authors used trichostatin A. The treatment for embryonic stem cells with trichostatin A over 6 or $10 \mathrm{~h}$ has proven to be efficient in obtaining chimeric mice in which a high percentage of specific tetramer-positive $\mathrm{CD} 8^{+} \mathrm{T}$ cells were observed in the absence of infection. Germline transmission was verified by backcrossing onto the MHC-matched mice (i.e., C57BL/6 and Balb/c background for T57 and G4R7, respectively). The authors used PCR and flow cytometry to identify offspring that carried the corresponding TCR. Thus, transgenic mice that presented three different TCR specificities, namely T57, G4 and R7 in the context of $\mathrm{H}-2 \mathrm{Ld}$ and $\mathrm{H}-2 \mathrm{~Kb}$, respectively, were obtained in a relatively short period of time ( $\sim 40$ days). Curiously, in MHC-I tetramer dissociation assay, transnuclear $(\mathrm{TN}) \mathrm{CD} 8^{+} \mathrm{T}$ cells

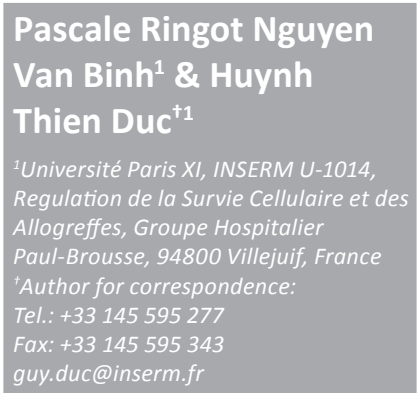

future medicine $^{\text {pits }} \mathbf{f S g}$ 
dissociated faster from their specific ligand than did cells from existing TCR transgenic mice (2C or OT-I). The authors argued that it could be possible that TCR from TN CD8 ${ }^{+}$ $T$ cells may be of low affinity because the effector cells were not highly selected in comparison with that of OT-I. Experiments concerning in vivo responses to $T$. gondii were investigated. It was observed that a high frequency $(60 \%)$ of specific effector $\mathrm{CD} 8^{+} \mathrm{T}$ cells was present in TN TCR mice compared with a percentage of $7 \%$ in wild-type mice after 9-16 days following parasite infection. When challenged with a lethal dose of $T$. gondii, four out of seven mice receiving $\mathrm{TN}$ T57 $\mathrm{CD} 88^{+} \mathrm{T}$ cells survived compared with one out of seven in controls without adoptive transfer. Using live imaging with luciferase-expressing pathogen, a significant reduction in $T$. gondii load was observed in the group receiving TN T57 CD8 ${ }^{+} \mathrm{T}$ cells compared with control.

\section{Discussion \& significance}

T-cell receptor transgenic mouse models are useful for studying functional aspects of the immune response. Unfortunately, only a few transgenic animals are available (i.e., ovalbumin-specific TCR transgenic OT-I, OT-II and DO11.10 mice). For studying the immune response in infectious disease, the availability of transgenic mice harboring TCRs specific to antigens from pathogens is essential. Kirak et al. reported a strategy using SCNT that allowed them to obtain transgenic mice with rearranged TCRs specific for three antigenic peptides of $T$. gondii. The technique used freshly isolated $\mathrm{CD} 8^{+} \mathrm{T}$ cells without genetic modification and appeared promising, as transgenic mice were obtained with apparent ease and in a relatively short time. According to the authors, some differences observed among SCNT TCR transgenic mice could be attributed to position effects within rearranged TCR genes and/or from a given expressed TCR. The protection was partial and limited when transgenic animals were submitted to lethal parasite challenge. This aspect raised questions regarding the nature and the type of donor cells, as well as the protective epitope. The low affinity of TN TCR for the relevant ligand is explained by the fact that donor cells were not highly selected. Overall, the work by Kirak et al. is interesting and could allow for the derivation of more rearranged TCR transgenic mice.

\section{Future perspective}

T-cell receptor transgenic mice represent an interesting model for exploring the functional aspect relevant to single clones that are, in general, difficult to study owing to their small number in wild-type organisms. The report by Kirak et al. described a new strategy for obtaining rearranged TCR transgenic mice by means of SCNT from freshly isolated effector $\mathrm{CD}^{+} \mathrm{T}$ cells. This strategy was not timeconsuming and generated transgenic mice without genetic modification. Although the presence of TN-specific effector $\mathrm{CD}^{+} \mathrm{T}$ cells reduced parasite burden, only limited protection to an acute lethal parasite challenge was observed. There are questions concerning the nature of the protective epitopes, as well as the stimulated cell type used as a donor for SCNT. Therefore, further experiments should explore these aspects, in other words, other cell types such as $\mathrm{CD}^{+}$ $\mathrm{T}$ cells, memory cells and perhaps $\mathrm{B}$ cells, that eventually could become an alternative method to hybridoma technology.

\section{Financial \& competing interests disclosure}

The financial support of this work is provided by Institut National de la Santé et de la Recherche Médicale (INSERM) U-1014, Regulation de la Survie Cellulaire et des Allogreffes, Université Paris XI, Groupe Hospitaler Paul-Brousse, 94800 Villejuif, France. The authors have no other relevant affliations or financial involvement with any organization or entity with a financial interest in or financial conflict with the subject matter or materials discussed in the manuscript apart from those disclosed.

No writing assistance was utilized in the production of this manuscript.

Executive summary

- CD8+ T cells are effector cytotoxic T lymphocytes that are programmed to exert lytic effects upon target cells expressing antigenic peptides presented by the relevant MHC-I.

- Somatic cell nuclear transfer is a technique that allows generation of embryonic stem cells and whole organisms by introduction of genetic material into enucleated oocytes.

- The paper under evaluation showed that it was possible to create TCR transgenic mice without genetic modification in a relatively short amount of time.

- The results hold promise for the generation of several other TCR transgenic mouse models against pathogens (bacteria and virus), allowing studies on the immunobiology of pathogen infection. Other studies relevant to fundamental aspects of immunoregulation could also take advantage of this technique. 


\section{Bibliography}

1 Jorgenson JL, Reay A, Ehrich W, Davis MM: Molecular components of $\mathrm{T}$ cell recognition. Ann. Rev. Immunol. 10, 835-873 (1992).

2 O'Shea JJ, Paul WE: Mechanisms underlying lineage commitment and plasticity of helper CD4+ T cells. Science 327, 1098-1102 (2010).

3 Harrington LE, van der Most R, Whitton JL et al:: Recombinant vaccinia virus-induced T-cell immunity: quantitation of the response to the virus vector and the foreign epitope. J. Virol. 76, 3329-3337 (2007).

4 He X-S, Rehermann B, Lopez-Labrador FX et al.: Quantitative analysis of hepatitis C virus-specific $\mathrm{CD}^{+} \mathrm{T}$ cells in peripheral blood and liver using peptide-MHC tetramers. Proc. Natl Acad. Sci. USA 96, 5692-5697 (1999).
5 Chen M, Sällberg M, Hughes J et al.: Immune tolerance split between hepatitis B virus precoce and core proteins. J. Virol. 79, 3016-3027 (2005).

6 Murphy KM, Heimberger AB, Loh DY: Induction by antigen of intrathymic apoptosis of $\mathrm{CD}^{+}{ }^{+} \mathrm{CD} 8{ }^{+} \mathrm{TCR}^{\text {lo }}$ thymocytes in vivo. Science 250, 1720-1723 (1990).

7 Barden MJ, Allison J, Health WR, Carbone FR: Defective TCR expression in transgenic mice constructed using cDNAbased $\alpha$ - and $\beta$-chain genes under the control of heterologous regulatory elements. Immunol. Cell Biol. 76, 34-40 (1998).

8 Scott CA, Paterson PA, Teyton L, Wilson IA: Crystal structures of two I-A D-peptide complexes reveal that high affinity can be achieved without large anchor residues. Immunity 8, 319-329 (1998).
9 Moudgil KD, Sercarz EE, Grewal IS: Modulation of the immunogenicity of antigenic determinants by their flanking residues. Immunol. Today 19, 217-220 (1998).

10 Robertson JM, Jensen PE, Evavold BD: DO11.10 and OT-II T cells recognize a C-terminal ovalbumin 323-339 epitope. J. Immunol. 164, 4706-4712 (2000).

11 Kirak O, Frickel EV, Grotenbreg GM et al.: Transnuclear mice with predefined $\mathrm{T}$ cell receptor specificities against Toxoplasma gondii obtained via SCNT. Science 238, 243-248 (2010). 